\title{
Correction: Relationship Between Gross Motor Function and Daily Functional Skill in Children With Cerebral Palsy
}

\author{
Tae Gun Kwon, $\mathrm{MD}^{1}$, Sook-Hee Yi, MD${ }^{1}$, Tae Won Kim, MS ${ }^{1}$, Hyun Jung Chang, $\mathrm{MD}^{2}$, Jeong-Yi Kwon, $\mathrm{MD}^{1}$
}

\author{
${ }^{1}$ Department of Physical and Rehabilitation Medicine, Samsung Medical Center, Sungkyunkwan University \\ School of Medicine, Seoul; ${ }^{2}$ Department of Physical Medicine and Rehabilitation, Samsung Changwon Hospital, \\ Sungkyunkwan University School of Medicine, Changwon, Korea
}

http://dx.doi.org/10.5535/arm.2013.37.1.41

Ann Rehabil Med 2013;37(1):41-49

To the Editor,

We found that a number of tables were inadvertently omitted. Summarized below are the additional information and the corresponding table numbers.

On page 44, line 12, 'Table 1' must be changed into 'Tables 4, 5.

On page 44, line 22, 'Table 1' must be changed into 'Table 4'.

On page 44 , line 32 , 'Table 1 ' must be changed into 'Table 5 .

On page 45 , line 9, 'Table 4' must be changed into 'Table 6.'

In Table 4, 'Table 4' must be changed into 'Table 6'

The following 2 tables (Tables 4 and 5) should appear in the manuscript.

Corresponding author: Jeong-Yi Kwon

Department of Physical and Rehabilitation Medicine, Samsung Medical Center, Sungkyunkwan University School of Medicine, 81 Irwon-ro, Gangnamgu, Seoul 135-710, Korea

Tel: +82-2-3410-2818, Fax: +82-2-3410-0052, E-mail: jeongyi@gmail.com

(c) This is an open-access article distributed under the terms of the Creative Commons Attribution Non-Commercial License (http://creativecommons.org/ licenses/by-nc/3.0) which permits unrestricted noncommercial use, distribution, and reproduction in any medium, provided the original work is properly cited.

Copyright ( 2013 by Korean Academy of Rehabilitation Medicine 
Table 4. GMFM-66 and PEDI-FSS stratified by GMFCS

\begin{tabular}{crllcc}
\hline \multirow{2}{*}{ GMFCS } & \multirow{2}{*}{ No. } & \multirow{2}{*}{ GMFM-66 } & \multicolumn{3}{c}{ PEDI-FSS } \\
\cline { 4 - 6 } & & & Self-care & Mobility & Social function \\
\hline I & 32 & $82.68 \pm 9.12^{\mathrm{b}, \mathrm{c}, \mathrm{d}, \mathrm{e})}$ & $70.90 \pm 8.71^{\mathrm{b}, \mathrm{c}, \mathrm{d}, \mathrm{e})}$ & $77.71 \pm 18.27^{\mathrm{b}, \mathrm{c}, \mathrm{d}, \mathrm{e})}$ & $73.55 \pm 13.26^{\mathrm{e})}$ \\
II & 31 & $64.81 \pm 8.44^{\mathrm{a}, \mathrm{c}, \mathrm{d}, \mathrm{e})}$ & $64.97 \pm 9.84^{\mathrm{d}, \mathrm{e})}$ & $61.98 \pm 13.65^{\mathrm{a}, \mathrm{c}, \mathrm{d}, \mathrm{e})}$ & $65.72 \pm 13.29^{\mathrm{e})}$ \\
III & 28 & $54.99 \pm 6.19^{\mathrm{a}, \mathrm{b}, \mathrm{d}, \mathrm{e})}$ & $62.17 \pm 11.60^{\mathrm{a}, \mathrm{d}, \mathrm{e})}$ & $50.43 \pm 11.62^{\mathrm{a}, \mathrm{b}, \mathrm{e})}$ & $70.60 \pm 16.20^{\mathrm{e})}$ \\
IV & 16 & $42.57 \pm 8.54^{\mathrm{a}, \mathrm{b}, \mathrm{c}, \mathrm{e})}$ & $52.23 \pm 10.61^{\mathrm{a}, \mathrm{b}, \mathrm{e})}$ & $38.33 \pm 6.78^{\mathrm{a}, \mathrm{b})}$ & $59.12 \pm 16.16^{\mathrm{e})}$ \\
V & 5 & $26.91 \pm 13.25^{\mathrm{a}, \mathrm{b}, \mathrm{c}, \mathrm{d})}$ & $34.60 \pm 12.27^{\mathrm{a}, \mathrm{b}, \mathrm{c}, \mathrm{d})}$ & $19.94 \pm 11.73^{\mathrm{a}, \mathrm{b}, \mathrm{c})}$ & $40.80 \pm 23.06^{\mathrm{a}, \mathrm{b}, \mathrm{c}, \mathrm{d})}$ \\
\hline Total & 112 & $62.59 \pm 17.76$ & $62.79 \pm 13.16$ & $58.33 \pm 20.96$ & $67.12 \pm 16.46$ \\
\hline
\end{tabular}

Values are presented as mean \pm standard deviation.

GMFM-66, Gross Motor Function Measure-66; PEDI-FSS, Pediatric Evaluation of Disability Inventory-Functional Skills Scale; GMFCS, Gross Motor Function Classification System.

p $<0.05$. Significantly different compared with ${ }^{\text {a) }}$ GMFCS I, ${ }^{\text {b) }}$ GMFCS II, ${ }^{\text {c) }}$ GMFCS III, ${ }^{\text {d) }}$ GMFCS IV, and ${ }^{\text {e) }}$ GMFCS V.

Table 5. GMFM-66 and PEDI-66 stratified by BFMF

\begin{tabular}{crllcc}
\hline \multirow{2}{*}{ BFMF } & \multirow{2}{*}{ No. } & \multirow{2}{*}{ GMFM-66 } & \multicolumn{3}{c}{ PEDI-FSS } \\
\cline { 4 - 6 } & & & Self-care & Mobility & Social function \\
\hline I & 6 & $72.18 \pm 15.08^{\mathrm{d}, \mathrm{e})}$ & $78.43 \pm 11.80^{\mathrm{c}, \mathrm{d}, \mathrm{e})}$ & $72.38 \pm 26.92^{\mathrm{d}, \mathrm{e})}$ & $82.95 \pm 14.17^{\mathrm{d}, \mathrm{e})}$ \\
II & 28 & $70.33 \pm 16.22^{\mathrm{d}, \mathrm{e})}$ & $66.42 \pm 7.19^{\mathrm{d}, \mathrm{e})}$ & $65.41 \pm 18.56^{\mathrm{d}, \mathrm{e})}$ & $69.01 \pm 13.65^{\mathrm{d}, \mathrm{e})}$ \\
III & 9 & $69.33 \pm 13.77^{\mathrm{d}, \mathrm{e})}$ & $60.91 \pm 8.48^{\mathrm{a}, \mathrm{d}, \mathrm{e})}$ & $70.00 \pm 16.68^{\mathrm{d}, \mathrm{e})}$ & $60.51 \pm 11.22^{\mathrm{e})}$ \\
IV & 10 & $42.82 \pm 10.35^{\mathrm{a}, \mathrm{b}, \mathrm{c})}$ & $46.88 \pm 12.41^{\mathrm{a}, \mathrm{b}, \mathrm{c}, \mathrm{e})}$ & $36.00 \pm 8.36^{\mathrm{a}, \mathrm{b}, \mathrm{c})}$ & $51.53 \pm 15.09^{\mathrm{a}, \mathrm{b})}$ \\
V & 2 & $14.69 \pm 9.28^{\mathrm{a}, \mathrm{b}, \mathrm{c})}$ & $23.80 \pm 3.39^{\mathrm{a}, \mathrm{b}, \mathrm{c}, \mathrm{d})}$ & $8.75 \pm 3.75^{\mathrm{a}, \mathrm{b}, \mathrm{c})}$ & $22.35 \pm 10.82^{\mathrm{a}, \mathrm{b}, \mathrm{c})}$ \\
\hline Total & 55 & $63.34 \pm 20.15$ & $61.73 \pm 14.56$ & $59.51 \pm 23.35$ & $64.26 \pm 17.84$ \\
\hline
\end{tabular}

Values are presented as mean \pm standard deviation.

GMFM-66, Gross Motor Function Measure-66; PEDI-FSS, Pediatric Evaluation of Disability Inventory-Functional Skills Scale; BFMF, Bimanual Fine Motor Function.

$\mathrm{p}<0.05$. Significantly different compared with ${ }^{\text {a) }}$ BFMF I, ${ }^{\text {b) }}$ BFMF II, ${ }^{\mathrm{c})}$ BFMF III, ${ }^{\mathrm{d})} \mathrm{BFMF}$ IV, and ${ }^{\mathrm{e})} \mathrm{BFMF}$ V.

We apologize for any inconvenience that is may have caused.

Yours sincerely, Jeong-Yi Kwon 